\title{
Smart Attendance Management System Using Face Recognition
}

\author{
K.V. Prasad Reddy ${ }^{1}$, R. Chaitanya Latha ${ }^{2}$, M. Lohitha², R. Sonia ${ }^{2}$, A. B. Usha ${ }^{2}$ \\ ${ }^{1}$ Associate Professor, Department of Computer Science and Engineering, Sanskrithi School of \\ Engineering, Puttaparthi, Andhra Pradesh, India \\ 2B.TECH. Scholar, Department of Computer Science and Engineering, Sanskrithi School ofEngineering, \\ Puttaparthi, Andhra Pradesh, India
}

\begin{abstract}
Article Info

Volume 8, Issue 3

Page Number : 706-711

\section{Publication Issue}

May-June-2021

\section{Article History}

Accepted : 10 June 2021

Published : 18 June 2021

To Maintain the attendance record with day to day activities is a challenging task. The conventional method of calling name of each student is time consuming and there is always a chance of proxy attendance. The smart attendance management will replace the manual method, which takes a lot of time consuming and difficult to maintain. There are many biometric processes, in that face recognition is the best method. Here we are using the computer vision which is a field of deep learning that is used for the camera reading and writing and using TkInter to create a GUI application.

Keywords: Attendance Management, Computer Vision, Deep Learning, TkInter.
\end{abstract}

\section{INTRODUCTION}

Biometrics are body measurements and calculations related to human characteristics. Biometrics authentication (or realistic authentication) is used in computer science as a form of identification and access control. It is also used to identify individuals in groups that are under surveillance.

Biometric identifiers are the distinctive, measurable characteristics used to label and describe individuals. Biometric identifiers are often categorized as physiological characteristics, which are related to the shape of the body. Examples include, but are not limited to fingerprint, palm veins, face recognition, DNA, palm print, hand geometry, iris recognition, retina and odor/scent. Behavioral characteristics are related to the pattern of behavior of a person, including but not limited to typing rhythm, gait, keystroke, signature, behavioral profiling, and voice. Some researchers have coined the term 'behaviometrics' to describe the latter class of biometrics.

\section{OBJECTIVE}

Traditionally, student's attendances during lecture session are taken manually by using attendance sheet given by the faculty in class, Which is a time consuming event. Moreover, it is very difficult to verify one by one student in a large classroom whether the authenticated students are actually 
responding or not. There are many biometric processes, in that face recognition is the best method. Here we are using the computer vision which is a field of deep learning that is used for the camera reading and writing and using TkInter to create a GUI application.

\section{EXISTING SYSTEM}

The model emphasize an existing method which is a scheme for recognizing the faces using Eigen face or Principal Component Analysis (PCA) methods. This is used for face recognition, detection and tracking. Here, PCA is based on the second order statistics of the image set, and does not address higher order statistical dependencies such as the relationships among three or more pixels. While PCA aims to find a second order correlation of patterns, Kernel PCA provides a replacement which takes into account higher order correlations. We compare the recognition results using kernel methods with Eigen face methods on two benchmarks.

\section{DRAWBACKS:}

It is Less accurate as compared to the proposed system and cannot be used properly for biometrics.

\section{PROPOSED SYSTEM}

In this paper, we proposed a method that which is able to take the attendance of a student by the face recognition of student. This paper is about the biometric attendance management. The automatic attendance management will replace the manual method, which takes a lot of time consuming and difficult to maintain. There are many biometric processes, in that face recognition is the best method. Here we are using the computer vision which is a field of deep learning that is used for the camera reading and writing and using TkInter to create a GUI application.

\section{ADVANTAGES:}

It is Accurate than compared to proposed system and it Increases productivity, Easy and safe to use and Convenient option.

\section{LITERATURE SURVEY}

[1] Naveed Khan Balcoh, M. HaroonYousaf, Waqar Ahma and M. Iram Baig: Students attendance in the classroom is very important task and if taken manually wastes a lot of time. There are many automatic methods available for this purpose i.e. Biometric attendance. All these methods also waste time because students have to make a queue to touch their thumb on the scanning device. This work describes the efficient algorithm that automatically marks the attendance without human intervention. This attendance is recorded by using a camera attached in front of classroom that is continuously capturing images of students, detect the faces in images and compare the detected faces with the database and mark the attendance. Summary: Students attendance in the classroom is very important task and if taken manually wastes a lot of time. There are many automatic methods available for this purpose i.e. Biometric attendance. All these methods also waste time because students have to make a queue to touch their thumb on the scanning device. This work describes the efficient algorithm that automatically marks the attendance without human intervention.

[2] NirmalayaKar, MrinalKanti Debbarma, Ashim Saha, and Dwijen RudraPal: Authentication is a significant issue in system control in computer based communication. Human face recognition is an important branch of biometric verification and has been widely used in many applications, such as video monitor system, human-computer interaction, and door control system and network security. This describes a method for Student's Attendance System 
which will integrate with the face recognition technology using Personal Component Analysis (PCA) algorithm. The system will record the attendance of the students in class room environment automatically and it will provide the facilities to the faculty to access the information of the students easily by maintaining a log for clock-in and clock- out time.

Summary: This describes a method for Student's Attendance System which will integrate with the face recognition technology using Personal Component Analysis (PCA) algorithm. The system will record the attendance of the students in class room environment automatically and it will provide the facilities to the faculty to access the information of the students easily by maintaining a log for clock-in and clock-out time.

[3] O. Shoewn: In this paper, the development of an attendance management system using biometrics is proposed. Managing student attendance during lecture periods has become a difficult challenge. The ability to compute the attendance percentage becomes a major task as manual computation produces errors, and also wastes a lot of time. For the stated reason, an efficient attendance management system using biometrics is designed. This system takes attendance electronically with the help of a finger print device and the records of the attendance are stored in a database. Attendance is marked after student identification.

Summary: For student identification, a biometric (fingerprint) identification based system is used. This process however, eliminates the need for stationary materials and personnel for the keeping of records. Eighty candidates were used to test the system and success rate of $94 \%$ was recorded. The manual attendance system average execution time for eighty students was 17.83 seconds while it was 3.79 seconds for the automatic attendance management system using biometrics.
[4] M. Turk and A. Pentland: An approach to the detection and identification of human faces is presented, and a working, near-real-time face recognition system which tracks a subject's head and then recognizes the person by comparing characteristics of the face to those of known individuals is described. This approach treats face recognition as a two-dimensional recognition problem, taking advantage of the fact that faces are normally upright and thus may be described by a small set of 2-D characteristic views. Face images are projected onto a feature space ('face space') that best encodes the variation among known face images. The face space is defined by the 'Eigen faces', which are the eigenvectors of the set of faces; they do not necessarily correspond to isolated features such as eyes, ears, and noses. The framework provides the ability to learn to recognize new faces in an unsupervised manner. Summary: This approach treats face recognition as a two-dimensional recognition problem, taking advantage of the fact that faces are normally upright and thus may be described by a small set of 2-D characteristic views. Face images are projected onto a feature space ('face space') that best encodes the variation among known face images. The face space is defined by the 'Eigen faces', which are the eigenvectors of the set of faces; they do not necessarily correspond to isolated features such as eyes, ears, and noses.

[5] Rekha A. L, Chethan H. K: The objective of this system is to present an automated system for human face recognition in a real time background for an organization to mark the attendance of their employees or student. So automated attendance using real time face recognition is a real world solution which comes with day to day activities of handling employees or student. The task is very difficult as the real time background subtraction in an image is still a challenge. In the past two decades, face detection and recognition has proven to be very interesting research field of image processing. 
Summary: The work carried out describes an automated attendance system using video surveillance. The proposed algorithm is automatic and efficient in intelligent surveillance applications. Video surveillance is used to detect the object movement thereby the captured image undergoes face detection and recognition process and searches the student database and enters the attendance if it is valid in the list.

\section{USE CASE DIAGRAM:}

Use case diagrams which are model behavior within a system and helps the developers to understand of what the user require. Its purpose is to present a graphical overview of thefunctionality provided by a system in terms of actors, their goals (represented as use cases), andany dependencies between those use cases. The main purpose of a use case diagram is to show what system functions are

performed for which actor. Roles of the actors in the system can be depicted. The stick man represents what's called an actor. Use case diagram can be useful forgetting an overall view of the system and clarifying who can do and more importantly what they can't do

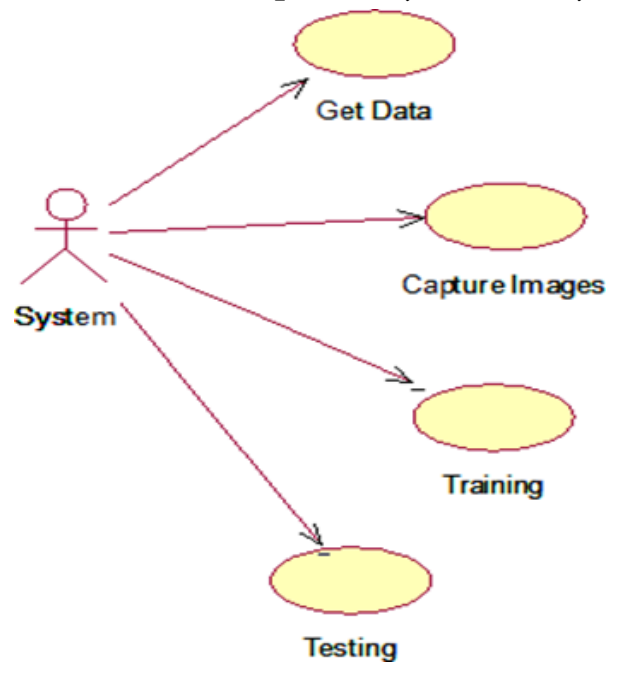

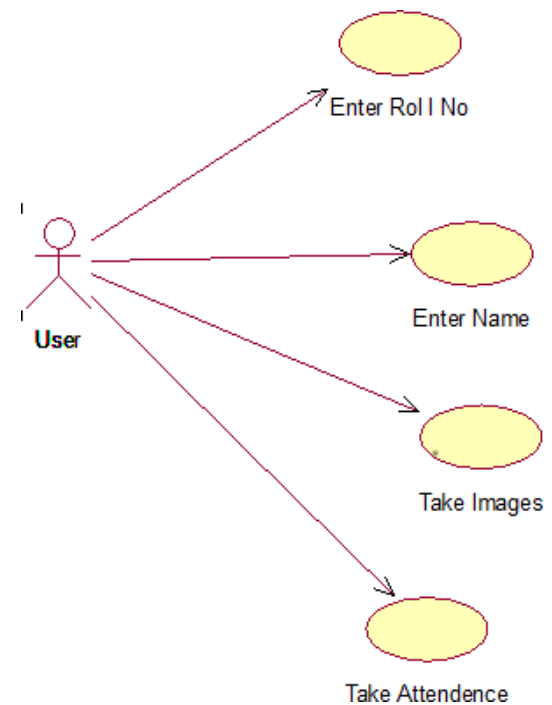

Use case diagram consists of use cases and actors and shows the interaction between the usecase and actors. SYSTEM REQUIREMENTS:

H/W Configuration:

Processor : I3/Intel Processor

Hard Disk : 160GB RAM : 8Gb

S/W Configuration:

Operating System : Windows 7/8/10 IDE : Pycharm. Libraries Used : Numpy, IO, OS,

TkInter.

Technology : Python 3.6+.

\section{BLOCK DIAGRAM}

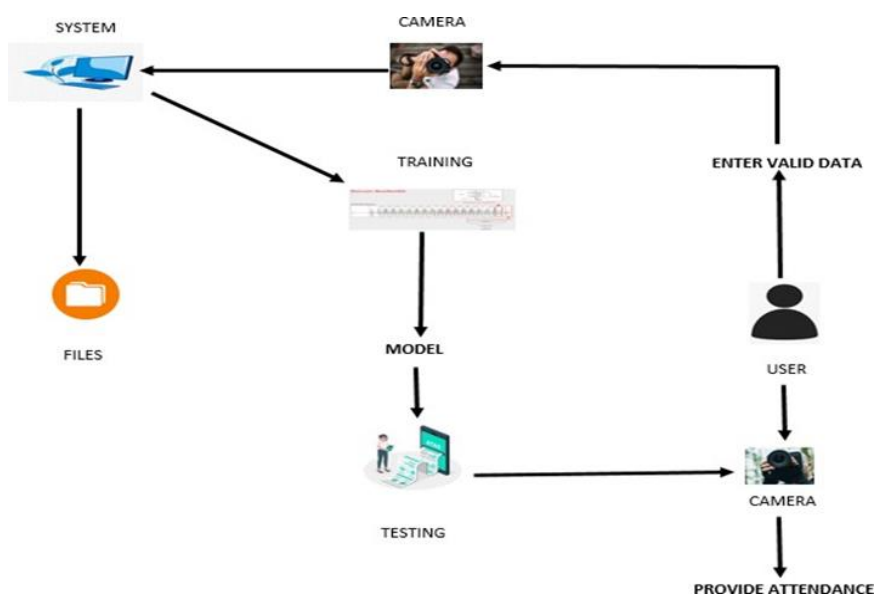


OUTPUT SCREENSHOTS: HOME PAGE:

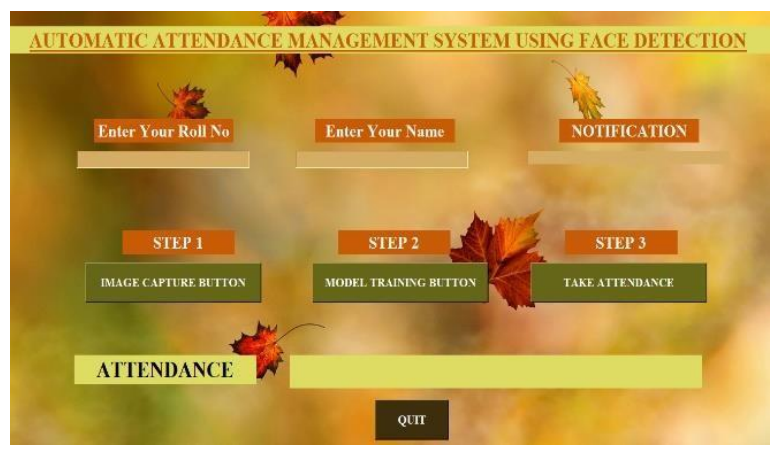

ENTER THE INFORMATION:

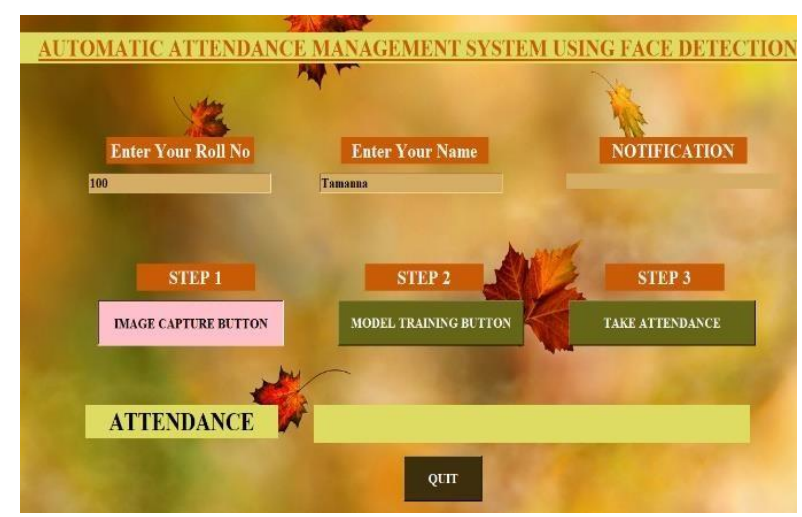

CAPTURE IMAGES:

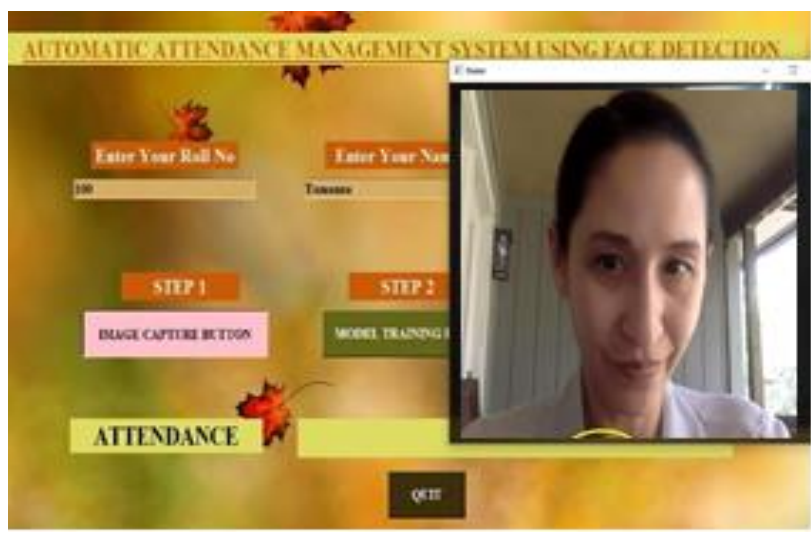

IMAGES CAPTURED SUCCESSFULLY:

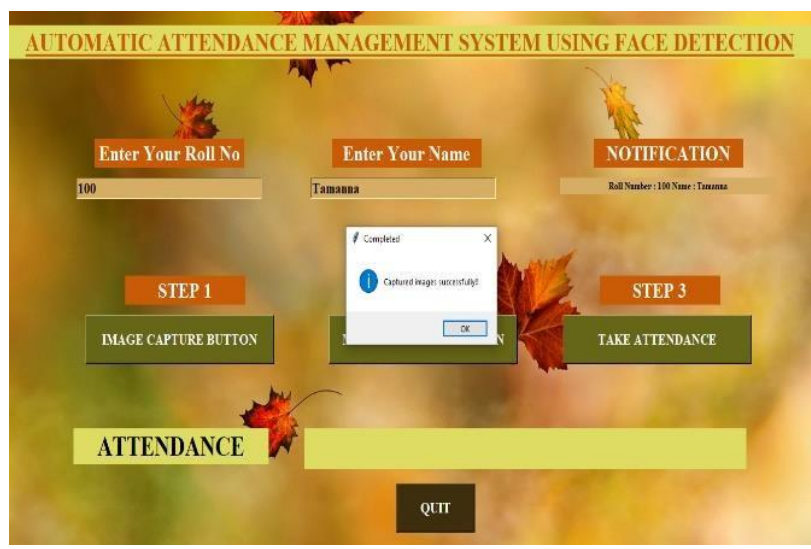

SUCCESSFULLY ATTENDANCE RECO DED:

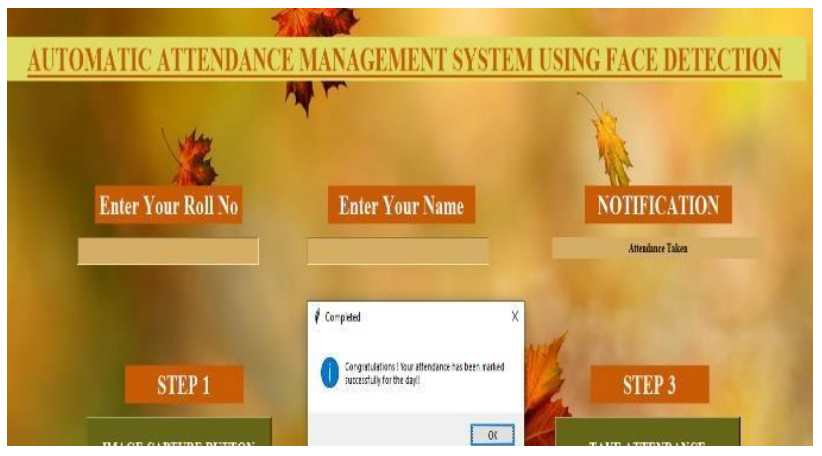

\section{CONCLUSION}

In our proposed work, we have created a model that which can take the attendance of student by the face recognition. We have used TkInter, where the information about the student are stored and a model is trained and then the student picture is captured which is tested and attendance is taken to the student by the captured face image.

\section{VII.FUTURE ENHANCEMENT}

In the future, we can extend the idea and can apply in different fields, like educational, corporate offices, and in many work places. That which can be easy to consider the attendance of a person with a lesser time and absenties list.

\section{REFERENCES}

[1]. EAI Endorsed Transactions on Creative Technologies Research Article on Smart Attendance Management System Using Face Recognition Kaneez Laila Bhatti, Laraib Mughal, Faheem Yar Khuhawar, Sheeraz Ahmed Memon Dept. of Telecommunication Engineering, MUET,2018.

[2]. Smart Attendance Management System Based On Face Recognition Algorithm by1M.Kasiselvanathan,2Dr.A.Kalaiselvi ,3Dr.S.P.Vimal,4V.Sangeet4Assistant Professor 
Sri Ramakrishna Engineering College, Coimbatore, Tamilnadu, India,2020.

[3]. Naveed Khan Balcoh, M. HaroonYousaf, Waqar Ahma and M. Iram Baig, Algorithm for efficient Attendance Management: Face Recognition Based approach, International Journal of Computer Science Issue, Vol.9, Issue 4, No 1, July 2012.

[4]. NirmalayaKar, MrinalKanti Debbarma, Ashim Saha, and Dwijen RudraPal, Study of implementing Automated Attendance System using Implementing Automated Attendance System Using face recognition Technique, International Journal of Computer and Communication Engineering, Vol 1, No 2,July 2012

[5]. O. Shoewn, Development of Attendance Management System using Biometrics. The Pacific Journal of Science and Technology Volume 13, No 1, May 2012

[6]. M. Turk and A. Pentland (1991) "Face recognition using Eigen faces". Proc.IEEE conference on computer vision and Pattern Recognition W.Zhao, R. Chellapa, P.J.Phillips and A.Rosenfld, "Face Recognition: A Literature Survey, vol. 35, No 4, Dec 2003, pp.399-458

[7]. R.L. Hsu, Mottalec M.A and A.K.Jain,"Face Detection in colour images", Proceedings International Conference on Image Processing, Oct 2001, pp. 1046-1049

[8]. ToufiqP. Ahmed Egammal and Anurag mittal (2006),"A Framework for feature selection for Background Subtraction", in Proceedings of IEEE computer Society Conference on Computer Vision and Pattern Recognition. M.H.Yang, N.Ahuja and D.Kriegmao, "Face recognition using kernel Eigen faces", IEEE International Conference on Image Processing, vol.1, pp. 10-13, Sept. 2000

[9]. Rekha A. L, Chethan H. K, “Automated Attendance System Management System Using
Face Recognition through Video Surveillance”, Volume 1, Issue 11, July- 2014

[10]. Anil K. Jain, Arun Ross and Salil Prabhakar, "An introduction to biometric recognition", Circuits and Systems for Video Technology, IEEE Transcations on Volume 14, Issu 1, Jan 2004 Page(s):4-20

[11]. H. M. El Barkey, "Face detection using fast neural networks and image decomposition", Neuro computing, Vol.11, no 3, pp 1039- 1046, 2002

[12]. R. L. Hsu, Mottale M.A and A.K. Jain, "Face detection in colour images", Proceedings International Conference on Image Processing (ICIP), Oct 2001, pp.1046-d

\section{Cite this article as :}

K. V. Prasad Reddy, R. Chaitanya Latha, M. Lohitha, R. Sonia, A. B. Usha, "Smart Attendance Management System Using Face Recognition", International Journal of Scientific Research in Science and Technology (IJSRST), Online ISSN : 2395-602X, Print ISSN : 2395-6011, Volume 8 Issue 3, pp. 706-711, May-June 2021. Available at doi : https://doi.org/10.32628/IJSRST2183162 Journal URL : https://ijsrst.com/IJSRST2183162 